\title{
The combined effect of physical activity and sedentary behavior on subclinical atherosclerosis: a cross-sectional study among Mexican Americans
}

Timothy J. Walker ${ }^{1 *}$ (D, Natalia I. Heredia ${ }^{2}$, MinJae Lee ${ }^{3}$, Susan T. Laing ${ }^{4}$, Susan P. Fisher-Hoch ${ }^{5}$, Joseph B. McCormick ${ }^{5}$ and Belinda M. Reininger ${ }^{6}$

\begin{abstract}
Background: Physical activity and sedentary behavior are considered independent risk factors for chronic disease. However, we do not fully understand their interrelation with key health outcomes such as subclinical atherosclerosis. This study examines the combined effects of sedentary behavior and physical activity on carotid intima-media thickness (cIMT) and presence of carotid plaque in a Mexican American population on the TexasMexico border.

Methods: This cross-sectional study was conducted using retrospective data from a sample $(n=612)$ of participants from the Cameron County Hispanic Cohort. Carotid ultrasound was used to measure CIMT and presence of carotid plaque. Self-reported questionnaires were used to assess leisure time physical activity and sedentary behavior (TV/ movie sitting and total sitting). A series of multivariable regression models were used to assess study aims. An interaction term between physical activity and sedentary behavior was included in models for each respective outcome. Models were controlled for demographic and health-related variables.

Results: There were no significant associations found between physical activity, sedentary behavior and mean cIMT, or CIMT thickness $\geq 75$ th percentile for age and gender. However, there was a significant interaction between physical activity and TV/movie sitting with presence of carotid plaque. Participants who reported moderate levels of physical activity had significantly lower odds for presence of plaque compared to participants with no activity when TV/movie sitting time was $\leq 3 \mathrm{~h}$ per day. However, there was no significant difference in odds for presence of plaque between physical activity groups when TV/movie sitting exceeded $3 \mathrm{~h} /$ day. These results were consistent with models examining total sitting time.

Conclusions: Our results indicate that for Mexican Americans, there is a combined effect of sedentary behavior and physical activity on presence of carotid plaque. Participating in moderate physical activity is optimal for having lower levels of carotid plaque in addition to avoiding excessive levels of TV/movie sitting ( $\geq 3 \mathrm{~h} /$ day) and/or total sitting ( $\geq 8.5 \mathrm{~h} /$ day).
\end{abstract}

Keywords: Physical activity, Sedentary behavior, Atherosclerosis, Carotid intima-media thickness, Carotid plaque

* Correspondence: Timothy.J.Walker@uth.tmc.edu

${ }^{1}$ Department of Health Promotion and Behavioral Sciences, Center for Health

Promotion and Prevention Research, The University of Texas Health Science

Center at Houston School of Public Health, 7000 Fannin St, Houston, TX

77030, USA

Full list of author information is available at the end of the article

(c) The Author(s). 2019 Open Access This article is distributed under the terms of the Creative Commons Attribution 4.0 International License (http://creativecommons.org/licenses/by/4.0/) which permits unrestricted use, distribution, and reproduction in any medium, provided you give appropriate credit to the original author(s) and the source, provide a link to the Creative Commons license, and indicate if changes were made. The Creative Commons Public Domain Dedication waiver (http://creativecommons.org/publicdomain/zero/1.0/) applies to the data made available in this article, unless otherwise stated. 


\section{Background}

Physical activity and sedentary behavior are considered distinct behaviors and independent risk factors for multiple health issues [1, 2]. Sedentary behavior is different from physical inactivity (insufficient levels of physical activity), where it has been defined as any waking behavior with an energy expenditure $\leq 1.5$ metabolic equivalents while in a sitting or lying position [3]. Research examining the health impact of sedentary behavior has grown rapidly. However, there is insufficient evidence to determine the combined effects that various levels of physical activity and sedentary behavior may have on key health outcomes including cardiovascular disease [1]. Much of the existing research assessing the interrelation between physical activity and sedentary behavior has focused on all-cause mortality and cardiovascular disease mortality [4]. Results from these studies provide evidence to support that the relation between sedentary behavior and mortality varies by the amount of physical activity [4].

Given the long-term health effects of physical activity and sedentary behavior, it is important to examine its combined effects with earlier stages of cardiovascular disease for preventive strategies. Subclinical atherosclerosis can be measured by carotid ultrasonography and multiple studies have shown prediction of cardiovascular events and stroke by carotid intima-media thickness (cIMT) and/ or presence of carotid plaque [5-10]. Presence of carotid plaque and cIMT are related, yet they capture different elements of cardiovascular health. As a result, these variables have been used independently or in an additive approach to predict cardiovascular risk [11].

Research examining the association between physical activity, sedentary behavior, and measures of subclinical atherosclerosis is less established where past studies in this area have reported mixed results [12-28]. These mixed findings could be a result of few studies assessing physical activity alongside sedentary behavior in adults $[17,19,20]$ or studies equating sedentary behavior to inactivity [27]. A better understanding of the potential combined effects of physical activity and sedentary behavior on subclinical atherosclerosis will provide needed clarity about the potential negative effect of sitting across different levels of activity for a critical health outcome.

Furthermore, to our knowledge, no study has assessed both the association of physical activity and sedentary behavior with cIMT or carotid plaque within a Mexican American population. Hispanics generally have lower levels of physical activity than other racial/ethnic groups [29, 30]. Given that Hispanics are the largest minority group in the U.S. and Mexican Americans are the largest sub-group of Hispanics [31], it is important to determine whether modifiable behaviors such physical activity and sedentary behavior are associated with subclinical atherosclerosis in a Mexican American population. Therefore, the purpose of this study is to assess the combined effects of both sedentary behavior (sitting time) and physical activity on cIMT and carotid plaque in Mexican Americans on the Texas-Mexico border.

\section{Methods \\ Study design and setting}

A cross-sectional design was used to assess study aims. Data for analyses were from the Cameron County Hispanic Cohort (CCHC), a randomly selected cohort of MexicanAmericans living in Brownsville, Texas, which is a homogenous population located on the US/Mexico border [32]. Extensive clinical data and self-reported questionnaire data capturing demographic and health-related information were collected from $\mathrm{CCHC}$ participants. The Institutional Ethics Review Board of the University of Texas Health Science Center at Houston approved this study.

\section{Participants}

Recruitment of cohort participants was completed by randomly selecting tract/blocks according to the 2000 Census as described in previous studies [32, 33]. Participants were 18 years or older and they were recruited from households where there could be multiple participants per household and from the same family. All participants gave written informed consent. Ongoing cohort data collection began in 2004, and a total of 4343 participants were considered for this analysis. In this study, we used de-identified data from a subset of $\mathrm{CCHC}$ participants who completed questionnaires and clinical studies relevant to research aims. Some clinical studies and questionnaires were introduced after the start of original data collection in 2004. Thus, data analyzed in this study were collected from March, 2010 to July, 2017.

\section{Measures \\ Carotid ultrasound evaluation}

Starting in 2010, cohort participants were invited to complete a Carotid ultrasound at their first visit or when they came in for a follow-up visit. Carotid ultrasounds were conducted using the Siemens Acuson X300 ultrasound system (Malvern, PA) using a VF 13-5 linear array transducer. Guidelines from the American Society of Echocardiography consensus statement on subclinical vascular disease were used to inform the study protocol [34]. Details of imaging procedures have been described elsewhere [35]. Primary study outcomes included cIMT thickness and presence of carotid plaque. Two variables were used to represent cIMT thickness: a continuous variable of thickness measured in millimeters and a dichotomous variable of having a cIMT thickness $\geq 75$ th percentile for age and gender. Presence of carotid plaque was defined as having wall thickening in the carotid artery that was $>50 \%$ of the thickness of the surrounding wall [36]. The selected 
measures of cIMT and carotid plaque are subclinical markers of early atherosclerosis and valid predictors of future cardiovascular events [5-10]. A single blinded expert read all images and measurements with replicate readings performed on $5 \%$ of the cohort with results indicating an intraclass correlation value of 0.96 .

\section{Physical activity and sedentary behavior variables}

The key independent variables were self-reported physical activity and time spent sitting watching TV/movies. Physical activity was measured using a modified version of the Godin Leisure-Time Exercise Questionnaire [37], the original version of which has been found to be a valid and reliable among Hispanics [38]. The physical activity questionnaire assessed participants' intensity, frequency, and duration of leisure time physical activity. Participants were categorized into four groups based on the volume (time and intensity) of weekly activity using standard metabolic equivalent (MET) values, where 1 MET is equivalent to the rate of energy expenditure at rest. For example, moderate intensity activity corresponds with 3.0-5.9 METs. Thus, a moderate activity of 4 METs performed for $150 \mathrm{~min}$ per week equates to 600 MET minutes/week [39], which is consistent with the amount of activity recommended by the US Physical Activity Guidelines for Americans [40]. The grouping of the physical activity variable was informed by the US Physical Activity guidelines: no activity (0 MET min/ wk), low activity (1-599 MET min/wk), moderate activity (600-1499 MET min/wk), and high activity ( $\geq 1500$ MET min/wk). Based on scoring protocols, participants were excluded from analyses if they reported extreme physical activity values ( $\geq 7680 \mathrm{MET} \mathrm{min} / \mathrm{wk}$ ).

Time spent watching $\mathrm{TV} /$ movies was originally assessed in the cohort survey using a single question asking participants to report the frequency and duration of sitting for TV or movies during the last 7 days. To improve the assessment of sedentary behavior, the multi-context sitting time questionnaire (MSTQ) was later added to the cohort survey as a more comprehensive measure with acceptable validity and reliability [41]. The MSTQ captures total sitting time in a typical weekand weekend day across the following domains: occupational, leisure-time, and transportation. Within the leisure-time domain, there is a question specific to sitting time while watching TV or movies. Therefore, sitting time for $\mathrm{TV} /$ movies was assessed by harmonizing the original survey question and the MSTQ question. More specifically, week- and weekend day TV/movie sitting (assessed by the MSTQ) were combined using a weighted average to capture total minutes per week of TV/movie sitting. The variable was then merged with the original $\mathrm{TV} /$ movie sitting question and rescaled to capture hours per day of $\mathrm{TV} /$ movie sitting to facilitate the interpretation of findings. A total sitting time (hours/day) variable was also created for the subsample of cohort participants who completed the MSTQ for a secondary analysis. The MSTQ includes a question about whether answers reflect a typical week of sitting where participants who responded "no" were excluded from analyses. Participants were also excluded if they reported daily sitting values $>24 \mathrm{~h}$.

\section{Covariates}

To control for confounding, variables from self-report questionnaires and laboratory studies were considered for inclusion in analyses based on their theoretical importance and previous research [35]. Demographic variables included gender, age, employment status (employed/unemployed), marital status (single/married, divorced/separated), and years of education. Smoking status was assessed by asking participants whether they smoke cigarettes. Alcohol was assessed by asking participants the number of drinks consumed per week (12 oz. beer, 8 oz. wine cooler, 4 oz. wine, or 1 oz. liquor). Drinks per week were converted to drinks per day where heavy alcohol use was considered to be more than two drinks per day for males and more than one drink per day for females [42]. Dietary patterns were assessed using the Healthy/Unhealthy Eating Indices where participants self-reported the number of times they ate a healthy (or unhealthy) food the previous day [43]. Healthy foods included fruit, fruit juice, baked/grilled poultry, baked/ grilled fish, whole grain breads, whole grain cereals, eggs, orange vegetables, salad, beans, and other vegetables where an index score was created from responses with a possible range from 0 to 55 . Unhealthy foods included regular sodas, sweetened drinks/sports drinks, sweetened tea, frozen desserts, pastries, non-chocolate candy, white bread, red and processed meats, fried meat, and French fries/chips where the index score could range from 0 to 50 . Having a history of stroke, heart attack, and angina (yes/no) were also assessed by a series of self-reported questions (e.g. have you ever been diagnosed or told by a doctor you had a stroke?).

Participants also completed a series of anthropometric measures and laboratory studies. Height and weight were measured to calculate body mass index (BMI). Systolic and diastolic blood pressure were measured by taking the average of the second and third readings occurring 5 minutes apart. Cohort participants provided blood samples for the following laboratory studies: fasting lipid panel (to assess cholesterol and triglycerides), glycated hemoglobin (HbA1c), fasting plasma glucose, and fasting serum insulin. To assess insulin resistance, the homeostasis model assessment insulin resistance index (HOMA-IR) was calculated using fasting glucose and insulin [44]. 


\section{Statistical analyses}

Descriptive statistics were assessed across the two dichotomous outcome variables. A multivariable linear regression model was used to determine whether physical activity and $\mathrm{TV} /$ movie sitting were significantly associated with mean cIMT. Two multivariable logistic regression models were used to determine whether physical activity and TV/movie sitting were significantly associated with 1) presence of plaque, and, 2) cIMT thickness $\geq 75$ th percentile. A first set of models only included the physical activity and sedentary behavior as independent variables. A next set of models adjusted for age and gender, and third set adjusted for age, gender, and additional demographic and health variables. The following demographic and health-related variables were considered for inclusion in models if they were statistically significant: employment status, marital status, years of education, healthy/unhealthy food indices, smoking status, heavy alcohol use, history of stroke, history of heart attack, history of angina, BMI, total cholesterol, triglycerides, systolic and diastolic blood pressure, HbA1c, and HOMA_IR. Models were also adjusted for having multiple participants from the same household by using generalized estimating equations (GEE) to control for clustering. An interaction term between physical activity and sedentary behavior was included in each model to assess whether the relation between sedentary behavior and outcomes were different across physical activity levels. A secondary analysis was also performed following the procedures previously described using the total sitting time variable with the subsample of participants who completed the MSTQ. All analyses were conducted using Stata 13.0.

\section{Results}

There were 727 cohort participants who completed a carotid ultrasound and self-report surveys that included the TV/ movie sitting questions. However, 95 participants were missing sedentary behavior data and 22 were excluded from analyses due to extreme values or reporting a non-normal week of sitting (Fig. 1). Thus, there were 612 participants with complete data for all three dependent and key independent variables from 459 unique households (Table 1). Some participants were missing data for other demographic and health-related control variables (Table 1) leading to sample sizes ranging from 592 to 607 in fully adjusted models. The majority of the sample was female (67.6\%) and middle-aged at the time of the ultrasound (mean age 50.4 \pm 14.1 years; Table 1). Most participants were not doing any leisure-time physical activity (64.4\% reported no physical activity) while the average time spent sitting for TV/movies was about $1.8 \mathrm{~h}$ per day. About $16 \%$ of participants had cIMT $\geq 75 \%$ while about $24 \%$ had presence of carotid plaque. There was a high prevalence of obesity (51.4\%) and other cardiovascular disease risk factors in the sample (32.4\% were hypertensive and $19.4 \%$ had diabetes). These health trends were similar when

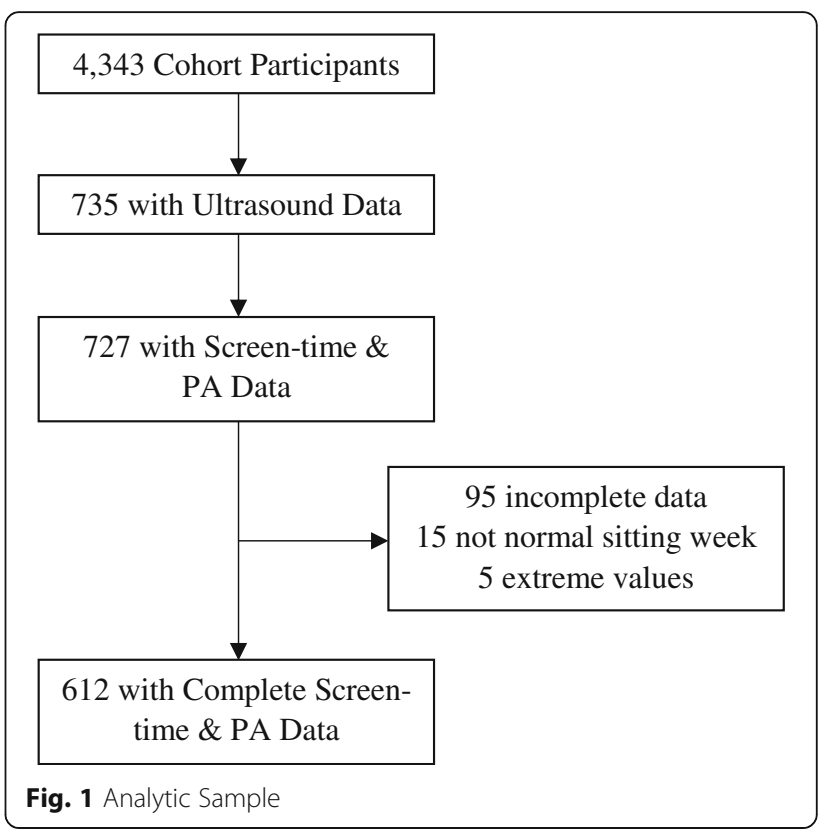

compared to the total cohort of participants $(n=4343)$ : obesity, 50.9\%; hypertension, 31.2\%; and diabetes, $20.2 \%$. However, in the complete cohort, there was a slightly lower percentage of females $(64.0 \%)$ and mean age $(45.3 \pm 17.2$ years).

\section{cIMT model results}

Results from the unadjusted linear model revealed a statistically significant inverse association between moderate and high levels of physical activity and mean cIMT (Table 2). However, after adjusting for age and gender, the associations between physical activity levels and mean cIMT decreased in value and were no longer statistically significant. There was no significant association between TV/movie sitting time and mean cIMT in the unadjusted or adjusted models (Table 2). Further, there was no evidence of an interaction between physical activity and TV/movie sitting time in relation to mean cIMT (results not shown). Results from models using the dichotomous cIMT $\geq 75 \%$ variable were relatively consistent with results from the linear cIMT models where there were no significant associations in unadjusted or adjusted models (Table 2), nor was there a significant interaction between physical activity and sedentary behavior (results not show).

\section{Carotid plaque model results}

Results from the unadjusted model revealed a statistically significant inverse associations between moderate and high physical activity levels and presence of carotid plaque, and, a statistically significant direct association between $\mathrm{TV} /$ movie sitting time and presence of carotid plaque (Table 2). Results from the age and gender adjusted, and fully adjusted models were mostly consistent 
Table 1 Descriptive statistics for total sample and across CIMT $\geq 75 \%$ and presence of carotid plaque variables

\begin{tabular}{|c|c|c|c|c|c|}
\hline \multirow[t]{2}{*}{ Variable, M (SD) } & \multirow{2}{*}{$\begin{array}{l}\text { Total } N= \\
612\end{array}$} & \multicolumn{2}{|l|}{$\mathrm{CIMT} \geq 75 \%$} & \multicolumn{2}{|c|}{ Presence of Carotid Plaque } \\
\hline & & Normal $N=512$ & Abnormal $N=100$ & No Plaque $N=462$ & Plaque $N=150$ \\
\hline \multicolumn{6}{|c|}{ Physical Activity and Sedentary Behavior Variables } \\
\hline \multicolumn{6}{|l|}{ Physical Activity (n, \%) } \\
\hline No Activity & $394(64.4)$ & $324(63.3)$ & $70(70.0)$ & $277(59.9)$ & $117(78.0)$ \\
\hline Low Activity & $49(8.0)$ & $44(8.6)$ & $5(5.0)$ & $39(8.4)$ & $10(6.7)$ \\
\hline Moderate Activity & $81(13.2)$ & $71(13.9)$ & $10(10.0)$ & $71(15.4)$ & $10(6.7)$ \\
\hline High Activity & $88(14.4)$ & $73(14.3)$ & $15(15.0)$ & $75(16.2)$ & $13(8.7)$ \\
\hline TV/Movie Sitting Time (hr./day) & $1.8(1.6)$ & $1.7(1.6)$ & $2.0(1.7)$ & $1.7(1.5)$ & $2.1(1.8)$ \\
\hline \multicolumn{6}{|l|}{ Demographic Variables } \\
\hline Female (n, \%) & $414(67.6)$ & $363(70.9)$ & $51(51.0)$ & $329(71.2)$ & $85(56.7)$ \\
\hline Age at cIMT & $50.4(14.1)$ & $49.5(14.5)$ & $54.9(10.6)$ & $46.9(13.5)$ & $61.0(9.9)$ \\
\hline Employed (n, \%) & $309(50.5)$ & $259(50.6)$ & $50(50.0)$ & $254(55.0)$ & $55(36.7)$ \\
\hline \multicolumn{6}{|l|}{ Marital Status (n, \%) } \\
\hline Single & $98(16.0)$ & $91(17.8)$ & $7(7.0)$ & 86 (18.6) & $12(8.0)$ \\
\hline Married & $406(66.3)$ & $333(65.0)$ & $73(73.0)$ & $304(65.8)$ & 102(68.0) \\
\hline Divorced/Separated & $108(17.7)$ & $88(17.2)$ & $20(20.0)$ & $72(15.6)$ & $36(24.0)$ \\
\hline Years Education & $11.1(5.5)$ & $11.3(5.6)$ & $10.1(4.7)$ & $11.5(5.4)$ & $9.8(5.5)$ \\
\hline \multicolumn{6}{|l|}{ Health Related Variables } \\
\hline \multicolumn{6}{|l|}{ Smoking (n, \%) } \\
\hline Never & $202(33.0)$ & $161(31.4)$ & $41(41.0)$ & $150(32.5)$ & $52(34.6)$ \\
\hline Sometimes & $135(22.1)$ & $110(21.5)$ & $25(25.0)$ & $93(20.1)$ & $42(28.0)$ \\
\hline Other & $275(44.9)$ & $241(47.1)$ & $34(34.0)$ & $219(47.4)$ & $56(37.3)$ \\
\hline Heavy Alcohol Use (n, \%) & $20(3.3)$ & $11(2.2)$ & $9(9.0)$ & $14(3.0)$ & $6(4.0)$ \\
\hline BMI (kg/m2) & $30.9(6.3)$ & $30.8(6.4)$ & $31.1(5.4)$ & $30.9(6.4)$ & $30.6(5.7)$ \\
\hline Total Cholesterol (mg/dL) & $182.4(37.9)$ & $181.1(36.4)$ & $188.9(44.5)$ & $181.0(35.2)$ & $186.8(45.0)$ \\
\hline Triglycerides (mg/dL) & $146.7(89.5)$ & $142.5(88.9)$ & $168.4(89.9)$ & $142.5(90.4)$ & $159.9(85.6)$ \\
\hline HDL-Cholesterol (mg/dL) & $45.9(13.2)$ & $46.3(13.0)$ & 43.9 (13.6) & $45.9(13.0)$ & $45.9(13.8)$ \\
\hline LDL-Cholesterol (mg/dL) & $108.0(31.8)$ & $107.2(30.7)$ & $112.1(36.8)$ & $107.2(30.3)$ & $110.5(36.0)$ \\
\hline Systolic Blood Pressure (mmHg) & $120.1(18.0)$ & $118.1(16.9)$ & $130.6(20.1)$ & $117.2(15.8)$ & $129.1(21.1)$ \\
\hline Diastolic Blood Pressure (mmHg) & $72.4(9.3)$ & $71.9(8.8)$ & $74.9(11.0)$ & $72.0(8.7)$ & $73.7(10.8)$ \\
\hline Insulin (mg/dL) & $13.1(9.7)$ & $13.0(9.7)$ & $13.6(9.9)$ & $13.0(9.4)$ & $13.4(10.5)$ \\
\hline Mean Glucose (mg/dL) & $116.9(38.0)$ & $112.6(33.4)$ & $138.1(50.6)$ & $111.5(31.9)$ & $133.5(49.1)$ \\
\hline HOMA_IR & $3.5(2.9)$ & $3.4(2.8)$ & $4.0(3.4)$ & $3.3(2.6)$ & $3.9(3.7)$ \\
\hline $\mathrm{Hba1C}(\%)$ & $5.8(1.3)$ & $5.7(1.22)$ & $6.4(1.5)$ & $5.7(1.2)$ & $6.3(1.5)$ \\
\hline Healthy Food Index & $7.2(3.6)$ & $7.2(3.5)$ & $7.4(3.8)$ & $7.1(3.6)$ & $7.4(3.5)$ \\
\hline Unhealthy Food Index & $4.7(3.2)$ & $4.7(3.2)$ & $4.8(3.2)$ & $4.8(3.3)$ & $4.4(2.7)$ \\
\hline History of Stroke $(n, \%)$ & $15(2.4)$ & $11(2.1)$ & $4(4.0)$ & $9(1.9)$ & $6(4.0)$ \\
\hline History of Heart Attack (n, \%) & $9(1.5)$ & $6(1.2)$ & $3(3.0)$ & $4(0.9)$ & $5(3.3)$ \\
\hline History of Angina (n, \%) & $10(1.6)$ & $8(1.6)$ & $2(2.0)$ & $5(1.1)$ & $5(3.3)$ \\
\hline
\end{tabular}

Cholesterol through Unhealthy Food Index had sample sizes ranging from 419 to 612

with the unadjusted model where there was a statistically significant inverse relation between moderate physical activity and presence of carotid plaque (Table 2). However, adjusted model results revealed non-significant trends for an inverse relation between high physical activity and presence of carotid plaque, and, a direct relation between $\mathrm{TV} /$ movie sitting time and presence of carotid plaque (Table 2). 
Table 2 Unadjusted and adjusted multivariable models with 4-category physical activity variable and TV/movie related sitting time

\begin{tabular}{|c|c|c|c|}
\hline & Mean difference of cIMT (95\% Cl) & CIMT $\geq 75 \%(O R, 95 \% \mathrm{Cl})$ & Presence of Plaque $(\mathrm{OR}, 95 \% \mathrm{Cl})$ \\
\hline \multicolumn{4}{|c|}{ Physical Activity (referent $=$ no activity) ${ }^{a}$} \\
\hline Low Activity & $-0.04(-0.08-0.001)$ & $0.51(0.18-1.47)$ & $0.64(0.31-1.31)$ \\
\hline Moderate Activity & $-0.05(-0.08--0.01)^{*}$ & $0.64(0.31-1.30)$ & $0.33(0.17-0.63)^{*}$ \\
\hline High Activity & $-0.06(-0.09--0.03)^{*}$ & $0.96(0.53-1.73)$ & $0.42(0.22-0.77)^{*}$ \\
\hline TV/Movie Sitting Time (hrs/day) & $0.001(-0.01-0.01)$ & $1.06(0.93-1.20)$ & $1.14(1.01-1.27)^{*}$ \\
\hline \multicolumn{4}{|c|}{ Physical Activity (referent $=$ no activity $)^{b}$} \\
\hline Low Activity & $-0.02(-0.04-0.01)$ & $0.53(0.18-1.54)$ & $0.74(0.34-1.62)$ \\
\hline Moderate Activity & $-0.02(-0.05-0.01)$ & $0.69(0.34-1.40)$ & $0.35(0.16-0.74)^{*}$ \\
\hline High Activity & $-0.01(-0.04-0.02)$ & $1.10(0.58-2.08)$ & $0.60(0.30-1.22)$ \\
\hline TV/Movie Sitting Time (hrs/day) & $-0.002(-0.01-0.01)$ & $1.02(0.90-1.16)$ & $1.10(0.97-1.25)$ \\
\hline \multicolumn{4}{|c|}{ Physical Activity (referent $=$ no activity) ${ }^{c}$} \\
\hline Low Activity & $-0.002(-0.03-0.02)$ & $0.51(0.13-1.95)$ & $0.94(0.42-2.08)$ \\
\hline Moderate Activity & $-0.009(-0.04-0.02)$ & $0.87(0.42-1.81)$ & $0.35(0.16-0.76)^{*}$ \\
\hline High Activity & $-0.009(-0.04-0.02)$ & $1.40(0.73-2.68)$ & $0.60(0.28-1.26)$ \\
\hline TV/Movie Sitting Time (hrs/day) & $-0.002(-0.01-0.005)$ & $0.97(0.85-1.14)$ & $1.10(0.96-1.25)$ \\
\hline
\end{tabular}

a, unadjusted model $(n=612) ; b$, adjusted for age and gender $(n=612) ; c$, fully adjusted model

Fully adjusted mean cIMT model included age, gender, cholesterol, systolic blood pressure, diastolic blood pressure and HBA1c $(n=593)$

Fully adjusted cIMT $\geq 75 \%$ model included age, gender, cholesterol, systolic blood pressure, heavy alcohol use, and HBA1c $(n=592)$

Fully adjusted plaque model included age, gender, cholesterol, and systolic blood pressure $(n=607)$

hrs/day is the weighted average of sitting on a typical weekday and weekend day

$*, p<0.05$

When testing the interaction between physical activity and $\mathrm{TV} /$ movie sitting, model results revealed a significant interaction suggesting the relation between physical activity and carotid plaque differed across levels of $\mathrm{TV} /$ movie sitting (Table 3). Participants who reported no physical activity had consistent odds for presence of plaque across all levels of TV/movie sitting. However, participants who reported moderate physical activity had lower odds for plaque across low levels of $\mathrm{TV} /$ movie sitting, but as $\mathrm{TV} /$ movie sitting time reached higher levels $(\geq 3 \mathrm{~h} /$ day $)$ the odds for plaque increased. When comparing odds between activity levels, participants who reported moderate levels of physical activity had significantly lower odds for presence of plaque compared to participants with no activity when TV/movie sitting time was $\leq 3 \mathrm{~h}$ per day (Table 3 ). However, when TV/movie sitting time exceeded $3 \mathrm{~h} /$ day, there was no significant difference in odds for presence of plaque between those who reported moderate activity versus those who reported no activity. Notably, for those who reported high activity levels, adjusted odds were relatively stable across all TV/movie sitting times with no statistically significant differences when compared to those with no activity.

\section{Secondary analysis}

Using total sitting time from the subsample of participants who completed the MSTQ led to a smaller analytic sample ranging from 336 to 340 participants in fully adjusted models. Results from these fully adjusted models were also similar to results from the primary analysis. There were no significant associations between physical activity and total sitting time with mean cIMT or cIMT $\geq 75 \%$ (Table 4). When assessing relations with plaque, there was a non-significant association between total sitting time and presence of carotid plaque while there was a significant inverse association

Table 3 Physical activity effect on carotid plaque across levels of TV/movie sitting time $(n=607)$

\begin{tabular}{lll}
\hline Physical activity x Sitting Time & Adjusted OR (95\% Cl) & $p$-value \\
\hline Low Activity vs No Activity & & \\
Sedentary at 0.33 h/day & $0.73(0.28-1.86)$ & 0.507 \\
Sedentary at 1.06 h/day & $0.83(0.37-1.87)$ & 0.662 \\
Sedentary at 1.96 h/day & $0.99(0.43-2.27)$ & 0.979 \\
Sedentary at 4.01 h/day & $1.46(0.34-6.14)$ & 0.610 \\
Moderate Activity vs No activity & & \\
Sedentary at 0.33 h/day & $0.19(0.07-0.55)$ & 0.002 \\
Sedentary at 1.06 h/day & $0.24(0.09-0.62)$ & 0.003 \\
Sedentary at 1.96 h/day & $0.32(0.13-0.75)$ & 0.009 \\
Sedentary at 4.01 h/day & $0.58(0.23-1.43)$ & 0.237 \\
High Activity vs No activity & & \\
Sedentary at 0.33 h/day & $0.65(0.22-1.85)$ & 0.416 \\
Sedentary at 1.06 h/day & $0.62(0.26-1.47)$ & 0.279 \\
Sedentary at 1.96 h/day & $0.59(0.29-1.23)$ & 0.163 \\
Sedentary at 4.01 h/day & $0.54(0.19-1.53)$ & 0.245 \\
\hline
\end{tabular}

Adjusted for age, gender, cholesterol, and systolic blood pressure 
between moderate physical activity and presence of carotid plaque (Table 4).

When testing the interaction between physical activity and total sitting time with the presence of plaque outcome, results were relatively consistent with the TV/ movie sitting time model (Table 5). Notably, participants who reported moderate physical activity had significantly lower odds for carotid plaque across lower levels of total sitting compared to participants who reported no activity (Table 5). However, at high levels of total sitting time $(\geq 8.5 \mathrm{~h} /$ day), there was no significant difference between adjusted odds of carotid plaque between participants who reported moderate activity to participants who reported no activity. Similar to the TV/movie sitting time model, there was no statistically significant difference in the adjusted odds for carotid plaque between participants who reported high activity versus participants who reported no activity across all levels of sitting time. However, there was a more prominent inverse trend for participants in the high activity group where a greater amount of total sitting was associated with lower adjusted odds for carotid plaque.

\section{Discussion}

This study examined the relations between physical activity, sedentary behavior, and subclinical atherosclerosis among a cohort of Mexican Americans. Results provide evidence that participating in moderate levels of physical activity are associated with lower odds for carotid plaque across low to moderate levels of sitting. However, at high levels of sitting, there appears to be no difference in odds for carotid plaque between physical activity levels. These results suggest participating in moderate physical activity and avoiding excessive levels of sitting time are associated with the lowest odds for carotid plaque compared to other combinations of physical activity and sedentary behavior. There was no evidence that physical activity or sedentary behavior were associated with mean cIMT (after adjusting for age and gender) among Mexican Americans.

Results were relatively consistent between the primary and secondary analyses. However, for those in the high physical activity group, a more prominent inverse trend between total sitting and adjusted odds for plaque appeared to be present in the secondary analysis. Because the secondary analysis was limited to those who completed the MSTQ, the study sample was reduced to 340 participants. This led to a low number of participants who had carotid plaque who were also moderately $(n=$ 3 ) or highly active $(n=6)$. This likely contributed to a more exaggerated inverse association in the highly active group than what was observed in the primary analysis. In addition, it may be why there was a more exaggerated increase in the odds of plaque for the moderate activity group at high values of sitting. Among participants who had complete sedentary behavior data, TV/movie sitting was moderately correlated with total sitting time $(\mathrm{r}=$ 0.40 ) and on average made up 35\% of total sitting time. Thus, TV/movie sitting is one of the more prominent sitting domains for this sample of Mexican Americans and likely served as an adequate proxy for total sitting time in this group.

Previous studies assessing the relation between physical activity and measures of subclinical atherosclerosis (cIMT and/or plaque) have reported inconsistent findings. Some studies have reported a significant inverse association between physical activity and cIMT [14, 15, 25, $27,45]$ while others have reported no association [13, $16,21,23$ ]. Results from a literature review [45] suggested inconsistent findings were likely due to a wide variability between study populations and weak measures of physical activity with the use of arbitrary cut-offs.

The lack of association between physical activity and cIMT in our study may be due to multiple factors. First, physical activity and sedentary behavior were measured at one time point capturing a participant's typical week of activity. This may not be representative of one's physical activity profile during adolescence or other periods of life, which may be an important factor in slowing the progression of atherosclerosis [23, 46, 47]. Second, the association between physical activity and mean cIMT was no longer significant after controlling for age and

Table 4 Adjusted multivariable GEE models with total sitting time (hrs/day)

\begin{tabular}{llll}
\hline & Adjusted Mean difference of cIMT $(95 \% \mathrm{Cl})$ & $\mathrm{cIMT} \geq 75 \%$ (adjusted OR, 95\% Cl) & Presence of Plaque (adjusted OR, 95\% Cl) \\
\hline Physical Activity (referent = no activity) & & $1.18(0.35-3.94)$ \\
Low Activity & $-0.009(-0.04-0.02)$ & $0.54(0.09-3.41)$ & $0.27(0.07-0.99)^{*}$ \\
Moderate Activity & $0.010(-0.03-0.05)$ & $1.17(0.45-3.07)$ & $0.41(0.14-1.15)$ \\
High Activity & $-0.014(-0.05-0.02)$ & $1.28(0.49-3.39)$ & $1.02(0.94-1.09)$ \\
Total Sitting Time (hrs/day) & $0.001(-0.003-0.004)$ & $0.95(0.89-1.02)$ & \\
\hline
\end{tabular}

cIMT model adjusted for age, gender, systolic blood pressure, diastolic blood pressure and HBA1c $(n=336)$

cIMT $\geq 75 \%$ model adjusted for age, gender, cholesterol, systolic blood pressure, and HBA1c ( $n=336)$

Plaque model adjusted for age, gender, and systolic blood pressure $(n=340)$

hrs/day is the weighted average of sitting on a typical weekday and weekend day

${ }^{*}, p<0.05$ 
Table 5 Physical activity effect on carotid plaque across levels of total sitting time $(n=340)$

\begin{tabular}{lll}
\hline Physical Activity x Sitting Time & Adjusted OR (95\% Cl) & p-value \\
\hline Low Activity vs No Activity & & \\
Sedentary at 1.99 h/day & $1.31(0.24-7.19)$ & 0.754 \\
Sedentary at 4.35 h/day & $1.25(0.32-4.82)$ & 0.750 \\
Sedentary at 7.68 h/day & $1.16(0.33-4.02)$ & 0.818 \\
Sedentary at 14.64 h/day & $0.99(0.09-10.52)$ & 0.995 \\
Moderate Activity vs No activity & & \\
Sedentary at 1.99 h/day & $0.03(0.002-0.35)$ & 0.005 \\
Sedentary at 4.35 h/day & $0.06(0.01-0.46)$ & 0.007 \\
Sedentary at 7.68 h/day & $0.18(0.36-0.85)$ & 0.030 \\
Sedentary at 14.64 h/day & $1.56(0.21-11.75)$ & 0.667 \\
High Activity vs No activity & & \\
Sedentary at 1.99 h/day & $1.50(0.27-8.49)$ & 0.645 \\
Sedentary at 4.35 h/day & $0.94(0.29-3.05)$ & 0.923 \\
Sedentary at 7.68 h/day & $0.49(0.18-1.31)$ & 0.155 \\
Sedentary at 14.64 h/day & $0.12(0.01-1.83)$ & 0.129 \\
\hline
\end{tabular}

Adjusted for age, gender, and systolic blood pressure

gender. Thus, cIMT may be driven by other risk factors and not physical activity and sedentary behavior levels $[5,7]$. Third, there was a relatively small number of people in respective physical activity groups who had a cIMT $\geq 75 \%$, which likely also contributed to the non-significant findings.

Our results did indicate a relation between physical activity and plaque even after controlling for other confounders. The association with plaque but not cIMT may be an indication that physical activity is associated with a more advanced stage of the atherosclerotic process. It is also possible that different risk factors are associated with cIMT and plaque suggesting they are related but not identical components in the development of atherosclerosis [5, 7, 48]. Studies indicate carotid plaque is related to atherosclerotic processes, which include inflammation, endothelial dysfunction, and oxidative stress whereas cIMT represents thickening of the smooth muscle and is primarily influenced by age and hypertension $[7,11,49]$. Thus, physical activity and sedentary behavior may be associated with plaque (and not cIMT) due to their influence on these atherosclerotic processes [50-52].

Notably, only those who participated in moderate (and not high) levels of physical activity had lower odds for plaque indicating physical activity and presence of plaque may not be linearly related. Other studies have reported a non-linear relation between physical activity and measures of atherosclerosis where it was suggested that very high levels of physical activity were associated with low-grade inflammation, which could exert an atherogenic effect $[23,53]$. It is also possible the non-linear trend we observed could be due to the relatively small sample size or limitations in using a self-report measure of physical activity, where people are known to over report activity levels [54], which may lead to some misclassifications in the physical activity categories.

Few studies have examined sedentary behavior as a risk factor for subclinical atherosclerosis where some studies have reported a direct association [14, 17] while others have reported no association [19, 20]. Sedentary behavior has been inconsistently operationalized throughout the literature (e.g., objectively measured, self-report, TV-sitting, inactivity, etc.) which is likely contributing to the inconsistent findings. To our knowledge, no studies have examined the interaction between physical activity and sedentary behavior to gain a better understanding of how the combined effect of these variables may be associated with measures of subclinical atherosclerosis. As previously reported, our results indicate moderate activity is associated with favorable outcomes. Our findings expand on the current literature by suggesting sitting time is not an additional risk factor for cIMT for people who are participating in no or low levels of physical activity. However, for those who participate in moderate physical activity, excessive levels of sitting time appear to contribute to higher odds for carotid plaque.

There are study limitations that need to be considered when interpreting results. First, this was a cross-sectional study that used self-report measures for physical activity and sedentary behavior. Even though the physical activity measure has been validated in a similar study population, we used a modified measure, which lacks additional validity testing. In addition, the sedentary behavior measure was changed during the study and later combined into single measures. Thus, we harmonized two different measures to capture TV/movie sitting time to maximize the study sample for the primary analysis. Further, the primary analysis was limited to $\mathrm{TV} /$ movie sitting, which serves as a proxy measure for total sitting levels. There are also some additional confounding factors we did not control for. For example, we did not take into account the potential impact of genetic risk on atherosclerosis and thus future studies should consider this factor. We also tried to control for diet using general variables to assess healthy and unhealthy eating, although results indicated the diet variables were not significantly contributing to models. A diet variable more focused on healthy eating to support cardiovascular health could have been more meaningful. Last, the majority of the sample was not physically active. Thus, there are a relatively small number of participants in the physical activity categories, which may impact the findings.

This study does have several strengths. First, it assessed relations among a homogeneous population of Hispanics. To our knowledge, this is the first study to examine 
physical activity and sedentary behavior with subclinical atherosclerosis among this population. In addition, our study focused on the interplay between physical activity and sedentary behavior to gain a better understanding about the activity profile rather than isolated, independent effects of sitting and physical activity. We also conducted a secondary analysis to assess total sitting time among a subsample of participants with a comprehensive measure of sedentary behavior. Results from the primary and secondary analyses were consistent providing additional support for findings. Lastly, even though we did not control for genetic risk and a specific diet to support cardiovascular health, analyses controlled for many potential confounders related to demographic, behavioral, and other health-related variables.

\section{Conclusions}

Promoting moderate physical activity and avoiding sedentary behavior needs to remain a public health priority given the numerous health benefits including its association with subclinical atherosclerosis. This is especially true among ethnic sub-groups, such as Mexican Americans, which is the largest sub-group of Hispanics in the US who often experience health disparities. Our results support that participating in moderate physical activity is optimal for having lower levels of carotid plaque in addition to avoiding excessive levels of total sitting ( $\geq 8.5 \mathrm{~h} /$ day) and/or TV/movie sitting ( $\geq 3 \mathrm{~h} /$ day). These findings are largely consistent with recommendations from the 2018 US Physical Activity Guidelines for Americans where adults are recommended to participate in at least 150 min of moderate aerobic activity per week and reduce levels of sitting [40]. However, we provide further evidence that when participating in moderate activity, health risks associated with sedentary behavior occur at very high levels of sitting per day. Thus, interventions focused on cardiovascular health can better help people achieve the associated health benefits by promoting a more focused message of $150 \mathrm{~min}$ of moderate aerobic activity per week and avoiding TV sitting for more than $3 \mathrm{~h}$ /day or total sitting for more than 8.5 $\mathrm{h} /$ day. Future research should continue to examine how the physical activity profile (both activity levels and sitting time) can impact other health outcomes such as coronary heart disease events and how genetics may play a role in these relationships.

\section{Abbreviations}

BMl: Body mass index; CCHC: Cameron County Hispanic Cohort: CIMT: Carotid intima-media thickness; GEE: Generalized estimating equations; HOMA-IR: Homeostasis model assessment insulin resistance index: MET: Metabolic equivalent; MSTQ: Multi-context sitting time questionnaire

\section{Acknowledgements}

The authors of this manuscript would like to acknowledge the participants who so willingly participated in this study, our community partners and Community Action Board members who are dedicated to eliminating health disparities. We would also like to acknowledge our Tu Salud ¡Si Cuenta! professional study team led by Lisa Mitchell- Bennett. We thank the cohort team, including Ms. Rocio Uribe and her team, who recruited and documented the participants. We also thank Marcela
Morris and other laboratory staff for their contributions, and Christina Villarreal for administrative support. Finally, we thank Valley Baptist Medical Center, Brownsville, Texas for providing us space for our Center for Clinical and Translational Science Clinical Research Unit.

\section{Funding}

The design of the cohort and data collection were funded by the EXPORT Grant from the National Center on Minority Health and Health Disparities (P20 MD000170), by the Center for Clinical and Translational Sciences (CCTS) mainly funded by the National Center for Advancing Translational Sciences (NCATS), and Centers for Translational Science Award (UL1 TR000371). The analysis, data interpretation, and writing were funded in part by the UTHealth School of Public Health Cancer Education and Career Development Program through a National Cancer Institute/NIH Grant (R25CA057712), the Center for Health Promotion and Prevention Research, the National Cancer Institute at the National Institutes of Health through The University of Texas MD Anderson's Cancer Center Support Grant (CA016672), a training grant from the Cancer Prevention and Research Institute of Texas (RP170259) and the Duncan Family Institute through the Center for Community-Engaged Translational Research. During the writing of the manuscript Dr. Walker was supported by a research career development award for (K12HD052023): Building Interdisciplinary Research Career in Women's Health ProgramBIRCWH; Berenson, PI) from the Eunice Kennedy Shriver National Institute of Child Health and Human Development (NICHD) at the National Institutes of Health. The content is solely the responsibility of the authors and does not necessarily represent the official views of the National Institutes of Health.

\section{Availability of data and materials}

The datasets used and/or analyzed during the current study are not publicly available due to privacy and ethics compliance but are available from the corresponding author on reasonable request.

\section{Authors' contributions}

TW and NH conceptualized the study. All authors made substantial contributions to the study design. TW and ML analyzed the data. SL, SFH, JM $\mathrm{ML}$, and BR made substantial contributions to the interpretation of data. TW and $\mathrm{NH}$ drafted the manuscript. All authors critically reviewed, edited, and approved the final manuscript.

\section{Ethics approval and consent to participate}

Study procedures were approved by The University of Texas Health Science Center at Houston Institutional Review Board. Written permission was obtained for access to CCHC data. Adults provided their written consent to participate.

\section{Consent for publication}

Not Applicable.

\section{Competing interests}

All authors declare they have no competing interests.

\section{Publisher's Note}

Springer Nature remains neutral with regard to jurisdictional claims in published maps and institutional affiliations.

\section{Author details}

'Department of Health Promotion and Behavioral Sciences, Center for Health Promotion and Prevention Research, The University of Texas Health Science Center at Houston School of Public Health, 7000 Fannin St, Houston, TX 77030, USA. ${ }^{2}$ Department of Health Disparities Research, The University of Texas MD Anderson Cancer Center, 1400 Pressler, Houston, TX 77030, USA. ${ }^{3}$ Division of Clinical and Translational Sciences, Department of Internal Medicine, The University of Texas McGovern Medical School; Biostatistics/ Epidemiology/Research Design (BERD) Core, Center for Clinical and Translational Sciences (CCTS), The University of Texas Health Science Center at Houston, 6410 Fannin St, Houston, TX 77030, USA. ${ }^{4}$ Division of Cardiology, Department of Internal Medicine, McGovern Medical School at the University of Texas Health Science Center at Houston, 6431 Fannin St, Houston, TX 77030, USA. ${ }^{5}$ Department of Epidemiology, Human Genetics and Environmental Sciences, The University of Texas Health Science Center at Houston School of Public Health, Brownsville Regional Campus, 1 W 
University Blvd, Brownsville, TX 78520, USA. ${ }^{6}$ Department of Health Promotion and Behavioral Sciences, The University of Texas Health Science Center at Houston School of Public Health, Brownsville Regional Campus, 1 W University Blvd, Brownsville, TX 78520, USA.

\section{Received: 17 August 2018 Accepted: 14 January 2019}

Published online: 06 February 2019

\section{References}

1. 2018 Physical Activity Guidelines Advisory Committee. 2018 Physical Activity Guidelines Advisory Committee Scientific Report. Washington, DC: US Department of Health and Human Services; 2018.

2. Gabriel KKP, Morrow JR, Woolsey A-LT. Framework for physical activity as a complex and multidimensional behavior. J Phys Act Heal. 2012;9:S11-8.

3. Tremblay MS, Aubert S, Barnes JD, Saunders TJ, Carson V, Latimer-Cheung AE, et al. Sedentary behavior research network (SBRN) - terminology consensus project process and outcome. Int J Behav Nutr Phys Act. 2017;14:75.

4. Ekelund U, Steene-Johannessen J, Brown WJ, Fagerland MW, Owen N, Powell $\mathrm{KE}$, et al. Does physical activity attenuate, or even eliminate, the detrimental association of sitting time with mortality? A harmonised meta-analysis of data from more than 1 million men and women. Lancet. 2016;388:1302-10.

5. Darabian S, Hormuz M, Latif MA, Pahlevan S, Budoff MJ. The role of carotid intimal thickness testing and risk prediction in the development of coronary atherosclerosis. Curr Atheroscler Rep. 2013;15(3):306.

6. Chambless LE, Heiss G, Folsom AR, Rosamond W, Szklo M, Sharrett AR, et al. Association of coronary heart disease incidence with carotid arterial wall thickness and major risk factors: the atherosclerosis risk in communities (ARIC) study, 1987-1993. Am. J. Epidemiol. 1997;146:483-94.

7. Inaba Y, Chen JA, Bergmann SR. Carotid plaque, compared with carotid intima-media thickness, more accurately predicts coronary artery disease events: a meta-analysis. Atherosclerosis. 2012;220:128-33.

8. Naqvi TZ, Lee MS. Carotid intima-media thickness and plaque in cardiovascular risk assessment. JACC Cardiovasc Imaging. 2014;7:1025-38.

9. Gepner AD, Young R, Delaney JA, Tattersall MC, Blaha MJ, Post WS, et al. Comparison of coronary artery calcium presence, carotid plaque presence, and carotid intima-media thickness for cardiovascular disease prediction in the multi-ethnic study of atherosclerosis. Circ. Cardiovasc. Imaging. 2015;1; 8(1):e002262

10. Wyman RA, Fraizer MC, Keevil JG, Busse KL, Aeschlimann SE, Korcarz CE, et al. Ultrasound-detected carotid plaque as a screening tool for advanced subclinical atherosclerosis. Am. Heart J. 2005;150(5):1081-1085

11. Amato M, Veglia F, de Faire U, Giral P, Rauramaa R, Smit AJ, et al. Carotid plaque-thickness and common carotid IMT show additive value in cardiovascular risk prediction and reclassification. Atherosclerosis. 2017; 263:412-9.

12. Folsom AR, Eckfeldt JH, Weitzman S, Ma J, Chambless LE, Barnes RW, et al. Relation of carotid artery wall thickness to diabetes mellitus, fasting glucose and insulin, body size, and physical activity. Atherosclerosis Risk in Communities (ARIC) Study Investigators. Stroke. 1994;25:66-73.

13. Ebrahim S, Papacosta O, Whincup P, Wannamethee G, Walker M, Nicolaides AN, et al. Carotid plaque, intima media thickness, cardiovascular risk factors, and prevalent cardiovascular disease in men and women: the British regional heart study. Stroke. 1999;30:841-50.

14. Parsons TJ, Sartini C, Ellins EA, Halcox JPJ, Smith KE, Ash S, et al. Objectively measured physical activity, sedentary time and subclinical vascular disease: cross-sectional study in older British men. Prev. Med. 2016;89:194-9.

15. Khalil A, Huffman MD, Prabhakaran D, Osmond C, Fall CHD, Tandon N, et al. Predictors of carotid intima-media thickness and carotid plaque in young Indian adults: the New Delhi birth cohort. Int. J. Cardiol. 2013;167:1322-8.

16. Gomez-Marcos MA, Recio-Rodríguez Jl, Patino-Alonso MC, Agudo-Conde C, Lasaosa-Medina L, Rodriguez-Sanchez E, et al. Relationship between objectively measured physical activity and vascular structure and function in adults. Atherosclerosis. 2014;234:366-72.

17. Diaz KM, Booth JN, Seals SR, Hooker SP, Sims M, Dubbert PM, et al. Sedentary behavior and subclinical atherosclerosis in African Americans: Cross-sectional analysis of the Jackson heart study. Int J Behav Nutr Phys Act. 2016;13:1-9.

18. Kozákova M, Palombo C, Morizzo C, Nolan JJ, Konrad T, Balkau B. Effect of sedentary behaviour and vigorous physical activity on segment-specific carotid wall thickness and its progression in a healthy population. Eur Heart J. 2010;31:1511-9.
19. García-Hermoso A, Martínez-Vizcaíno V, Recio-Rodríguez Jl, Sánchez-López M, Gómez-Marcos MÁ, García-Ortiz L. Sedentary behaviour patterns and carotid intima-media thickness in Spanish healthy adult population. Atherosclerosis. 2015;239:571-6.

20. Kronenberg F, Pereira MA, Schmitz MK, Arnett DK, Evenson KR, Crapo RO, et al. Influence of leisure time physical activity and television watching on atherosclerosis risk factors in the NHLBI family heart study. Atherosclerosis. 2000;153:433-43.

21. Lakka TA, Laukkanen JA, Rauramaa R, Salonen R, Lakka HM, Kaplan GA, et al. Cardiorespiratory fitness and the progression of carotid atherosclerosis in middle-aged men. Ann Intern Med. 2001;134:12-20.

22. Luedemann J, Schminke U, Berger K, Piek M, Willich SN, Doring A, et al. Association between behavior-dependent cardiovascular risk factors and asymptomatic carotid atherosclerosis in a general population. Stroke. 2002;33:2929-35.

23. Kwaśniewska M, Jegier A, Kostka T, Dziankowska-Zaborszczyk E, Rębowska E, Kozińska J, et al. Long-Term Effect of Different Physical Activity Levels on Subclinical Atherosclerosis in Middle-Aged Men: A 25-Year Prospective Study. PLoS One. 2014;9:e85209.

24. Spring B, Moller AC, Colangelo LA, Siddique J, Roehrig M, Daviglus ML, et al. Healthy lifestyle change and subclinical atherosclerosis in young adults: coronary artery risk development in young adults (CARDIA) study. Circulation. 2014;130:10-7.

25. Stensland-Bugge E, Bønaa KH, Joakimsen O, Njølstad I. Sex differences in the relationship of risk factors to subclinical carotid atherosclerosis measured 15 years later : the Tromsø study. Stroke. 2000;31:574-81.

26. Bertoni AG, Whitt-Glover MC, Chung H, Le KY, Barr RG, Mahesh M, et al. The association between physical activity and subclinical atherosclerosis: the multi-ethnic study of atherosclerosis. Am J Epidemiol. 2009;169:444-54.

27. Nordstrom CK, Dwyer KM, Merz CNB, Shircore A, Dwyer JH. Leisure time physical activity and early atherosclerosis: the Los Angeles atherosclerosis study. Am J Med. 2003;115:19-25.

28. Pahkala K, Heinonen OJ, Simell O, Viikari JS, Rönnemaa T, Niinikoski H, et al. Association of physical activity with vascular endothelial function and intima-media thickness. Circulation. 2011;124:1956-63.

29. August KJ, Sorkin DH. Racial/ethnic disparities in exercise and dietary behaviors of middle-aged and older adults. J Gen Intern Med. 2011;26:245-50.

30. Marquez DX, Neighbors CJ, Bustamante EE. Leisure time and occupational physical activity among racial or ethnic minorities. Med Sci Sports Exerc. 2010;42:1086-93.

31. Flores A. How the U.S. Hispanic population is changing [Internet]. Pew Res. Cent. 2017 [cited 2017 Dec 1]. Available from: http://www.pewresearch.org/ fact-tank/2017/09/18/how-the-u-s-hispanic-population-is-changing/

32. Fisher-Hoch SP, Rentfro AR, Salinas JJ, Pérez A, Brown HS, Reininger BM, et al. Socioeconomic status and prevalence of obesity and diabetes in a Mexican American community, Cameron County, Texas, 2004-2007. Prev Chronic Dis. 2010;7:1-10.

33. Fisher-Hoch S, Vatcheva K, Laing S, Hossain M, Rahbar M, Hanis C. Missed Opportunities for Diagnosis and Treatment of Diabetes, Hypertension, and Hypercholesterolemia in a Mexican American Population, Cameron County Hispanic Cohort, 2003-2008. Prev Chronic Dis 2012;9.

34. D'Agostino RB, Vasan RS, Pencina MJ, Wolf PA, Cobain M, Massaro JM, et al. General cardiovascular risk profile for use in primary care: the Framingham heart study. Circulation. 2008;117:743-53.

35. Laing ST, Smulevitz B, Vatcheva KP, Rahbar MH, Reininger B, McPherson DD, et al. Subclinical atherosclerosis and obesity phenotypes among Mexican Americans. J Am Heart Assoc 2015;4:e001540.

36. Stein JH, Korcarz CE, Hurst RT, Lonn E, Kendall CB, Mohler ER, et al. Use of carotid ultrasound to identify subclinical vascular disease and evaluate cardiovascular disease risk: a consensus statement from the American Society of Echocardiography carotid intima-media thickness task force endorsed by the Society for Vascular. J Am Soc Echocardiogr. 2008 Feb 1; 21(2):93-111.

37. Godin G, Shephard RJ. Godin Leisure-Time Exercise Questionnaire. Med Sci Sports Exerc. 1997;29:36-8.

38. Rauh MJ, Hovell MF, Hofstetter CR, Sallis JF, Gleghorn A. Reliability and validity of self-reported physical activity in Latinos. Int J Epidemiol. 1992;21:966-71.

39. Bauman A, Bull F, Chey T, Craig CL, Ainsworth BE, Sallis JF, et al. The International Prevalence Study on Physical Activity: results from 20 countries. Int. J. Behav. Nutr. Phys. Act. 2009;6:21.

40. U.S. Department of Health and Human Services. Physical Activity Guidelines for Americans, 2nd edition. Washington, DC: U.S. Department of Health and Human Services; 2018. 
41. Whitfield GP, Gabriel KKP, Kohl HW. Assessing sitting across contexts: development of the multicontext sitting time questionnaire. Res Q Exerc Sport. 2013;84:323-8.

42. Reininger BM, Wang J, Fisher-Hoch SP, Boutte A, Vatcheva K, McCormick JB. Non-communicable diseases and preventive health behaviors: a comparison of Hispanics nationally and those living along the US-Mexico border health behavior, health promotion and society. BMC Public Health. 2015;15(1):564.

43. Heredia NI, Lee MJ, Mitchell-Bennett L, Reininger BM. Tu Salud iSí Cuenta! Your Health Matters! A Community-wide Campaign in a Hispanic Border Community in Texas. J Nutr Educ Behav. 2017;49:801-809.

44. Matthews DR, Hosker JP, Rudenski S, Naylor B, Treacher DF, Turner RC Homeostasis model assessment: insulin resistance and beta-cell function from fasting plasma glucose and insulin concentrations in man. Diabetologia. 1985;28(7):412-9.

45. Kadoglou NPE, lliadis F, Liapis CD. Exercise and Carotid Atherosclerosis. Eur. J. Vasc. Endovasc. Surg. 2008;35(3):264-72.

46. Palatini P, Puato M, Rattazzi M, Pauletto P. Effect of regular physical activity on carotid intima-media thickness. Results from a 6-year prospective study in the early stage of hypertension. Blood Press. 2010;20:37-44.

47. Lima MCS, Barbosa MF, Diniz TA, Codogno JS, Freitas Júnior IF, Fernandes RA. Early and current physical activity: relationship with intima-media thickness and metabolic variables in adulthood. Brazilian J Phys Ther. 2014;18:462-9.

48. Bonithon-Kopp C, Touboul PJ, Berr C, Leroux C, Mainard F, Courbon D, et al. Relation of intima-media thickness to atherosclerotic plaques in carotid arteries: the vascular aging (EVA) study. Arterioscler Thromb Vasc Biol. 1996;16:310-6.

49. Kwaśniewska M, Kostka T, Jegier A, Dziankowska-Zaborszczyk E, Leszczyńska J, Rębowska E, et al. Regular physical activity and cardiovascular biomarkers in prevention of atherosclerosis in men: a 25-year prospective cohort study. BMC Cardiovasc. Disord. 2016;16:65.

50. Sallis JF, Saelens BE. Assessment of physical activity by self-report: status, limitations, and future directions. Res Q Exerc Sport. 2000;71:1-14.

51. Finn AV, Kolodgie FD, Virmani R. Correlation between carotid intimal/medial thickness and atherosclerosis: a point of view from pathology. Arterioscler Thromb Vasc Biol. 2010;30(2):177-81

52. Warburton DER, Nicol CW, Bredin SSD. Health benefits of physical activity: the evidence. Canadian medical association journal. 2006;174(6):801-9.

53. Kasapis C, Thompson PD. The effects of physical activity on serum Creactive protein and inflammatory markers: a systematic review. J Am Coll Cardiol. 2005:45(10):1563-9.

54. Ertek S, Cicero A. Impact of physical activity on inflammation: effects on cardiovascular disease risk and other inflammatory conditions. Arch Med Sci. 2012 Nov 9:8(5):794

Ready to submit your research? Choose BMC and benefit from:

- fast, convenient online submission

- thorough peer review by experienced researchers in your field

- rapid publication on acceptance

- support for research data, including large and complex data types

- gold Open Access which fosters wider collaboration and increased citations

- maximum visibility for your research: over $100 \mathrm{M}$ website views per year

At $\mathrm{BMC}$, research is always in progress.

Learn more biomedcentral.com/submissions 